\section{An investigation of the effect of two types of sentence contexts on paired-associate learning}

\author{
SHEILA M. PFAFFLIN \\ Bell Telephone Laboratories, Murray Hill, N.J. 07974
}

Two experiments investigated the use of sentences as mnemonic devices in paired-associate learning. The first experiment was concerned with the effect of adjectival modification of nouns presented in sentences on noun recall. The second experiment investigated the question of whether "bizarre" sentences would be particularly effective as mnemonics. Contrary to expectation from a visual imagery interpretation of sentence mnemonics, adjectives depressed recall and the less bizarre sentences were more effective mnemonics.

The two experiments described in this paper investigate the use of sentences as mnemonic devices in paired-associate learning. Mnemonic systems commonly stress the importance of vivid, bizarre images as mnemonic devices (Yates, 1966). Various investigators have hypothesized that visual imagery inderlies memory for sentences made up of concrete words (Begg \& Paivio, 1969; Wearing, 1971) and is involved in the facilitation of paired-associate learning by sentence mnemonics (Reese, 1970; Levin, 1970). If these views are correct, the more vivid and bizarre the imagery induced by a sentence, the greater its mnemonic effect should be. Adjectives might be expected to augment the vividness of the imagery induced by sentences, so the first experiment investigated their effect by comparing recall of noun pairs from sentences in which adjectives modified the nouns with recall of unmodified nouns in sentences, and with a no-sentence condition.

The second experiment investigated the properties of the verbs used to connect noun pairs presented in sentences to see whether their "bizarreness" was related to their effectiveness in facilitating learning. The sentence equivalent for a "bizarre" image is uncertain. Presumably, however, it would be a sentence expressing a relation unlikely to occur between the two nouns in question. Conversely, a relation which is likely to occur between two nouns should not be "bizarre." In this experiment, therefore, sentences using verbs likely to relate the nouns were compared with a condition in which the same verbs were randomly reassigned to the noun pairs to provide bizarre sentences. A third condition, in which the related verbs were replaced by verbs similar in meaning but infrequently selected to relate the nouns, was included in an effort to determine whether the response frequency of the specific word used to express the relation was important.

\section{EXPERIMENT 1}

\section{Design}

All Ss received the three types of stimulus material: word pairs (WP), simple active sentences in which the nouns were not modified (SP), and simple active sentences in which the nouns were modified (MSP). The noun pairs were divided into three sets and were assigned to the three stimulus conditions using a Latin-square design so that the effect of individual pairs was counterbalanced for the experimental treatments. Order of stimulus and response presentation was randomized for different groups of Ss.

Ss were 15 Technical Assistants (TAs), Senior Technical Assistants (STAs), and Associate Members of the Technical Staff (AMTSs) employed at the Murray Hill, New Jersey, location of the Bell Telephone Laboratories. The educational backgrounds of members of this population range from some college or technical school training to Master's degrees.

\section{Stimuli}

The basic stimuli consisted of 24 noun pairs. The nouns, consisting of object names with Thorndike-Lorge (1944) frequencies ranging from 17 to over 100 occurrences per million words, were paired randomly. The modification sentence frame consisted of "The (adjective) (noun) (verb) the (adjective) (noun)." Examples of the sentences used are "The wide arch hid the tiny insect" and "The old truck crushed the big snake." The same sentences, without adjectives, were used in the sentence-without-modification condition. The noun appearing to the left served as the stimulus noun. The stimulus and response nouns were underlined in the sentence conditions.

\section{Procedure}

The study-test method of

\section{Subjects}

paired-associate learning was used. The task was described to $\mathrm{Ss}$, and they were told to expect the various kinds of stimulus materials. The experimental materials were presented to the Ss using a Revere $35-\mathrm{mm}$ slide projector. They had 2.5 sec per slide on both study and test trials, with a 2.3 -sec interslide interval and an intertrial interval of $30 \mathrm{sec}$. Study and test trials alternated, starting with a study trial. On the test trials, only the stimulus nouns were shown, and the Ss wrote down the response nouns that they recalled. The Ss served in groups of two to five for five trials.

$$
\text { Results }
$$

Two analyses of variance were done, one on the total errors, summed for all trials, and one on the errors for the first trial only. No effects involving the conditions of stimulus presentation were significant in the analysis of total errors. In the analysis of errors from the first learning trial, the main effect associated with the different learning conditions was significant $[F(2,24)=5.06, p<.025]$. The mean number of first trial errors for the three conditions were: $W P=5.27$, $\mathrm{SP}=6.20, \mathrm{MSP}=6.80$. The standard error for these means, calculated from the mean square within-s error variance, is 0.30 . The differences between the means were examined by the Newman-Keuls test, which indicated that the WP-MSP difference is significant at the .05 level. The other differences were not significant.

\section{EXPERIMENT 2}

\section{Design}

In this experiment, recall of nouns from sentences with relevant frequent (HRF) verbs, relevant infrequent (LRF) verbs, and irrelevant (IR) verbs were compared in a within-S design. The noun pairs were formed into three groups that were counterbalanced by use of a Latin-square design across the experimental conditions. The same random orders were used for different groups so that order of presentation within trials was partially confounded with sentence group and counterbalanced for the main effect of the experimental conditions.

\section{Stimuli}

The verbs that were used with these noun pairs were obtained from a study in which 50 Ss were asked to supply the verb they considered most likely to relate the two nouns in a simple active sentence. From a somewhat larger set of noun pairs (the 24 noun pairs used in Experiment 1) for which these data were obtained, a set of 18 was then selected (some pairs had to be eliminated, chiefly because of duplication of the primary verb). The verbs were then used either in sentences with the noun pair for which they were the dominant verb or with 
some other noun pair to form the IR and RHF conditions. The low-frequency related condition was formed by replacing the high-frequency related verbs with verbs that were similar in meaning but that seldom occurred as responses in the study from which the high-frequency verbs were obtained. In the case of three pairs, no low-frequency response of similar meaning was available, and synonyms were supplied by the $E$. The median frequency of occurrence in the preliminary study for HRF verbs was 14 , with a range from 29 to 6 ; for the LRF verbs, the median was 1 , with a range from 6 to 0 . It should also be noted that while verbs of any Thorndike-Lorge (T-L) frequency could have been used as responses, the high communality responses show the usual tendency of having high T-L frequencies, while the infrequent responses tend to have lower $\mathrm{T}-\mathrm{L}$ frequencies. As a result, the HRF and LRF verbs differ in median $T-L$ frequency, as well as in their frequency of occurrence as responses to these noun pairs. The median T-L frequency for RHF verbs exceeds 100 per million (AA); that for the RLF verbs is 19 per million. Examples of the sentences used, with the verbs employed in the various conditions shown separated by slashes, are: "The screw injured (HRF) / lacerated (LRF) / lit (IR) the man" and "The glove covered (HRF) / concealed (LRF) / climbed (IR) the leaf."

\section{Subjects}

Ss were 30 TAs, STAs, and AMTSs employed at the Murray Hill, New Jersey, location of the Bell Telephone Laboratories.

\section{Procedure}

The study-test method of paired-associate learning was used, starting with a study trial and alternating test and study trials thereafter. On test trials, only the stimulus noun (the first noun in the sentence) was presented, and the S's task was to supply the other noun from the sentence. The learning materials were presented to Ss individually. First, instructions were read to the $\mathrm{S}$ explaining the task. The stimuli were presented on slides by means of a Mast random access projector controlled by a DDP 116 computer. The criterion for learning was one errorless trial for all Ss. Different random orders were used for each stimulus and stimulus/response series. The slides of both series were shown for $3.0 \mathrm{sec}$ with a 2.5-sec interval between slides. Ss wrote their responses on response trials. There was a 10-sec interval between trials during which an instruction slide appropriate to the particular series was presented. Results

An analysis of variance was performed upon the number of errors made by Ss under the various conditions. A significant main effect associated with the conditions of stimulus presentation was found $[F(2,54)=5.56, p<.01]$. The mean number of errors per $S$ for the three conditions were: $L R F=7.27$, $\mathrm{HRF}=7.77, \mathrm{IR}=9.73$. The standard error for the means, calculated from the mean square within-S error variance, is 0.55 . The differences between these means were analyzed by Tukey's Gap test, which indicated a difference between the mean for IR condition and the means for other two conditions significant at the .02 level. The LRF and HRF means did not differ significantly.

\section{GENERAL DISCUSSION}

The results of these two experiments do not provide much support for the vivid, bazarre imagery explanation of the mnemonic effect of sentences on paired-associate learning. Interpretation of the results of the first experiment is complicated by the finding that the sentence context alone did not facilitate learning. While such absence of facilitation is not unknown (Levin, 1970; Stolurow \& Bergum, 1958), the conditions that determine the effect of sentence mnemonics are not yet clear; a possibility in the present experiment is that the use of a within-S design with sentence and nonsentence stimuli may have created conditions unfavorable to the effective processing of the sentences. Since the effect of adjective modification may interact with such conditions, the result might have been different in sentence contexts that were themselves facilitating. However, so far as the sentences and conditions used in this experiment go, one must either argue that the adjectives actually reduce the effectiveness of sentence-induced imagery or assume that they hinder recall in some other way, perhaps by increasing the disparity between training and test stimuli, for example.

The results of the second experiment are at variance with a bizarre-relation interpretation of sentence mnemonic effects. The most effective mnemonics were those relations that were considered most likely to occur between the two nouns. Such a result is not predicted by a theory that considers bizarre relations more facilitating than common relations but is consistent with the view that the associative strength of the relation determines its effectiveness. These results thus seem most consistent with the view that relations that have a high likelihood of being spontaneously elicited by the items to be remembered make the best mnemonic devices.

\section{REFERENCES}

BEGG. I., \& PAVIO. A. Concreteness and imagery in sentence meaning. Joumal of Verbal Learning \& Verbal Behavior. $1969,8,821-827$

LEVIN, J. Factors related to the sentence facilitation of paired-associate learning. Joumal of Educational Psy chology, 1970, 61, 431-439.

REESE H. W. Imagery and contextual meaning. Psychological Bulletin, 1970, $73,404-414$.

STOLUROW, L. M. \& BERGUM. B. Leaming diagnostic information-elfects of direction of association and of prose of direction of association and of prose Finch and F. Cameron (Eds.). Symposium on Air Force human engineering personnel and training research Washington, D.C: National Academy of Sciences-National Research Council, Publication 516, 1958. Pp. 69-84.

THORNDIKE, $\mathrm{E}$ \& LORGE, I. The teacher's word book of 30.000 words. New York: Bureau of Publications. Teachers College, Columbia University, 1944.

WEARING, A. J. Vividness in the recall of English nominalizations. Psychonomic Science, 1971, 22, 121-122.

YATES, F. The art of memory. Chicago: University of Chicago Press, 1966. 